Article

\title{
Activity and Export of Engineered Nisin-(1-22) Analogs
}

\author{
Annechien Plat, Anneke Kuipers, Jacobien G. de Lange, Gert N. Moll * and Rick Rink \\ BiOMaDe Technology Foundation, Nijenborgh 4, 9747 AG Groningen, The Netherlands; \\ E-Mails: plat@biomade.nl (A.P.); kuipers@lanthiopep.nl (A.K.); ja.g.de.lange@gmail.com (J.G.L.); \\ rink@lanthiopep.nl (R.R.) \\ * Author to whom correspondence should be addressed; E-Mail: moll@lanthiopep.nl; \\ Tel.: +31-50-363-8070; Fax: +31-50-363-4429.
}

Received: 4 July 2011; in revised form: 29 July 2011 / Accepted: 11 August 2011 /

Published: 12 August 2011

\begin{abstract}
The pentacyclic peptide antibiotic nisin, produced by Lactococcus lactis is ubiquitously applied as a food preservative. We previously demonstrated that the truncated nisin-(1-22) has only 10-fold lower activity than nisin. Here we aimed at further developing this tricyclic nisin analog to reach activity comparable to that of nisin. Our data demonstrate that: (1) ring A has a large mutational freedom; (2) the composition of residues 20-22 strongly affects production levels of nisin-(1-22); (3) a positively charged C-terminus of nisin-(1-22) significantly enhances its antimicrobial activity; (4) nisin-(1-22) inhibits in vitro growth of a target strain using different dynamics than nisin.
\end{abstract}

Keywords: nisin; lantibiotic; peptide; cyclization; Lactococcus

\section{Introduction}

The increasing incidence of bacterial resistance to antibiotics is a worldwide problem. Hence developing new antibiotics and improving the ones already available are highly important. Understanding the structures and mechanisms of known antibiotics is a vital part of these processes. An interesting candidate antibiotic is the antimicrobial peptide nisin A (Figure 1) [1]. Although nisin has been widely used for a long time as a food-preservative in a broad range of products [2,3], resistance is limited [4,5]. Nisin is produced by Lactococcus lactis and exerts antimicrobial activity against a broad range of Gram-positive bacteria. It consists of 21 unmodified amino acids, 
2 dehydroalanines, 1 dehydrobutyrine, 1 lanthionine and 4 methyllanthionines. Nisin is ribosomally synthesized as a prepeptide of 57 amino acids, consisting of an N-terminal leader sequence and a $\mathrm{C}$-terminal propeptide sequence $[1,6]$. The serine and threonine residues in the propeptide sequence are dehydrated by NisB into dehydroalanine and dehydrobutyrine, respectively. The cyclase NisC subsequently catalyzes the coupling reaction between the double bond in the dehydroamino acids and the thiol group of cysteines. The resulting (methyl) lanthionine rings make nisin a member of a group of antibiotics called lantibiotics [7]. The fully modified prenisin is transported across the cytoplasmic membrane by the ABC-type transporter NisT where it is extracellularly activated by NisP-mediated proteolytic removal of the leader sequence [6]. Nisin induces NisRK activity which in turn induces transcription of the biosynthetic and immunity genes. L. lactis protects itself against nisin via lipoprotein NisI, which probably acts as an intercepting molecule, and the ABC-transporter NisFEG.

Figure 1. Nisin A. Dha, dehydroalanine; Dhb, dehydrobutyrine; Ala-S-Ala, mesolanthionine; Abu-S-Ala, $\beta$-methyllanthionine.

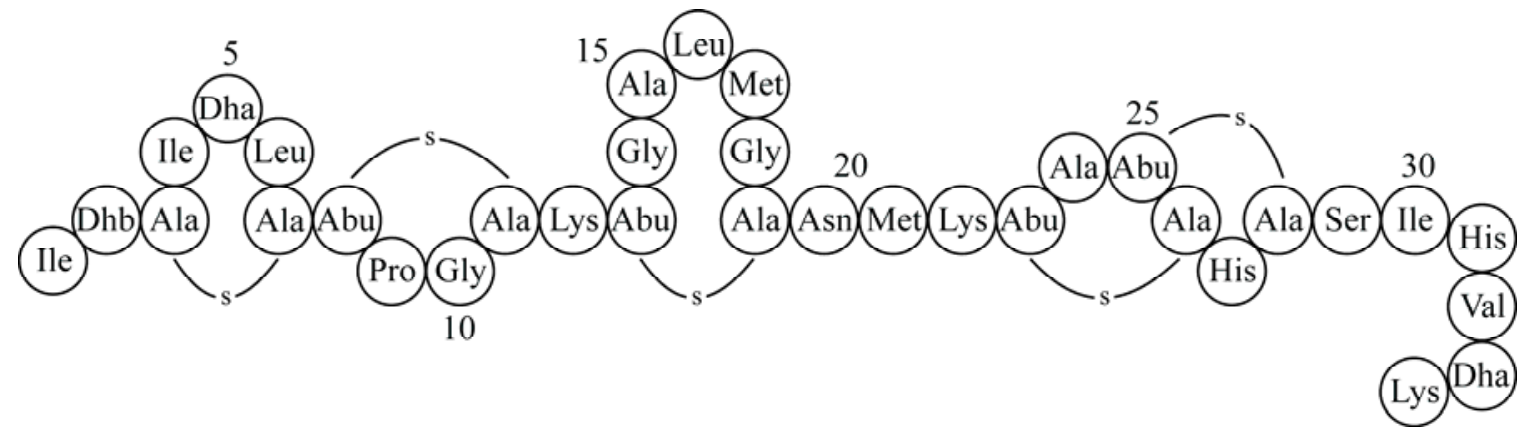

We previously demonstrated that by randomization of ring A of nisin, a large number of mutants could be generated, among which mutants with enhanced activity against specific target organisms [8]. However, ring A mutations which led to improved antimicrobial activity of full length nisin did not necessarily imply an improved activity of nisin-(1-22). Therefore we here applied randomization of ring $\mathrm{A}$ in nisin-(1-22) itself. In addition, we randomized the C-terminal tail of truncated nisin-(1-22).

Studies on the activity of truncated nisin mutants showed that nisin-(1-32)- $\mathrm{NH}_{2}$, nisin-(1-29) and nisin-(1-20) have respectively slightly higher, about 10-fold lower and 100-fold lower activity than nisin [9]. Rink et al. found that nisin-(1-22) has only 10-fold lower activity than nisin and suggested that the positively charged Lys22 might enhance binding to the negatively-charged bacterial membrane [8]. Analogously, the elimination of the carboxyl charge of nisin-(1-32)- $\mathrm{NH}_{2}$ might account for its slightly higher activity than that of nisin. In this study we tested the activity and export of a series of nisin-(1-22) analogs with a cationic or anionic tail, or randomized tail. Truncated nisin analogs with a cationic or anionic tail were obtained by engineering additional C-terminal lysines or $\mathrm{C}$-terminal aspartates. Furthermore, one negative charge was eliminated by $\mathrm{C}$-terminal amidation.

Nisin inhibits cell wall synthesis by abduction of lipid II from the septum [10]. A second mechanism of antimicrobial activity is constituted by the formation of hybrid pores in the target cell membrane composed of both nisin and lipid II [11-14]. By contrast nisin-(1-22) does not form pores [8]. Hence its antimicrobial activity likely results solely from the abduction of lipid II which leads to inhibition of cell wall synthesis. To gain further mechanistic insight we here investigated in vitro the dynamics of mixtures of nisin and nisin-(1-22). 


\section{Experimental Section}

Lactococcus lactis NZ9000 was used for expression of the modification enzymes and prenisin constructs. L. lactis was grown in M17 broth [15] supplemented with $0.5 \%$ glucose (GM17) or in minimal medium [16,17] with or without chloramphenicol $(\mathrm{Cm}, 5 \mu \mathrm{g} / \mathrm{mL})$ and/or erythromycin (Em, $5 \mu \mathrm{g} / \mathrm{mL}$ ). Prior to analysis of peptides produced in the media, cells were cultured as follows. Overnight cultures of L. lactis NZ9000, grown in GM17 broth containing antibiotics, were diluted 1/100 in minimal medium. Production of prenisin, prenisin-(1-22) and prenisin-(1-22) analogs was induced by adding supernatant of L. lactis NZ9700 1:1000, containing approximately $1 \mathrm{mg} / \mathrm{L}$ wild type nisin. Strains and plasmids are listed in Table 1.

Table 1. Bacterial strains and plasmids.

\begin{tabular}{|c|c|c|}
\hline Strain or plasmid & Characteristics & Reference \\
\hline \multicolumn{3}{|l|}{ Strain } \\
\hline Lactococcus lactis NZ9000 & $n i s R K^{+}$ & [18] \\
\hline L. lactis NZ9700 & nisABTCIPRKEFG & [18] \\
\hline L. lactis LL108(pORI 280) & $\mathrm{Em}^{\mathrm{r}} \mathrm{Cm}^{\mathrm{r}}$ & {$[18,19]$} \\
\hline L. lactis $\mathrm{pNGnisT} \mathrm{pNGnisP}$ & nisTP & [20] \\
\hline \multicolumn{3}{|l|}{ Plasmid } \\
\hline pNZnisA-E3 & Nisin A & [20] \\
\hline pNZ-E3 trWT 5 & Nisin-(1-22) & [8] \\
\hline pIL3BTC & nisBTC & [17] \\
\hline
\end{tabular}

Standard genetic manipulations were performed using established procedures [21]. Constructs coding for truncated prenisin analogs were made by amplifying plasmid pNZnisA-E3 [20] via round-PCR using a downstream sense primer and an upstream antisense primer. Each primer pair contained one 5' phosphorylation and a (non-annealing) peptide-encoding tail. The DNA amplification was performed with Phusion DNA polymerase (Finnzymes, Finland). Self-ligation of the resulting linear plasmids was carried out with T4 DNA ligase (Roche, Mannheim, Germany). The resulting plasmids were transformed into L. lactis NZ9000 containing pIL3BTC which encodes the modification and transport enzymes. Electrotransformation of L. lactis was carried out as previously described using a Bio-Rad gene pulser (Richmond, CA) [22]. Nucleotide sequence analysis was performed by BaseClear (Leiden, Netherlands).

Prenisin, prenisin-(1-22) and prenisin-(1-22) analogs were purified from culture supernatant. Minimal medium culture supernatant was diluted with an equal volume of $100 \mathrm{mM}$ lactic acid (pH 4). The prepeptides were subsequently purified by a single passage of the supernatant over a $5 \mathrm{~mL}$ HiTrap SP Sepharose cation-exchange column (GE Healthcare). Elution was performed at pH 4.0 with $1 \mathrm{M}$ $\mathrm{NaCl}$ in $50 \mathrm{mM}$ lactic acid. The fraction containing the prepeptide was desalted on a PD10 column (GE Healthcare) and subsequently lyophilized. After removal of the leader peptide, nisin and derived analogs were further purified via reversed-phase high-performance liquid chromatography (HPLC) on a $\mathrm{C} 18$ column with a gradient of 10 to $50 \%$ acetonitrile in $0.1 \%$ trifluoroacetic acid. The purified peptides were quantified on the basis of a comparison of the $214 \mathrm{~nm}$ HPLC peak area with that of wild type nisin. 
The leader peptide was cleaved off by incubating the prepeptides with $0.02 \mathrm{mg} / \mathrm{mL}$ trypsin for $30 \mathrm{~min}$ to 1 hour at $37{ }^{\circ} \mathrm{C}$. However, incubating truncated prenisin analogs containing additional $\mathrm{C}$-terminal lysines with trypsin led to removal of these engineered $\mathrm{C}$-terminal lysines. We previously demonstrated that alternative cleavage sites can be engineered in the C-terminal part of the nisin leader without interfering with the leader peptide's capacity to induce posttranslational modifications [23]. In the truncated prenisin analogs containing additional lysines, ASPR was replaced by ASPE via mutagenesis. The R-1E mutation allows cleavage with the protease Glu-C which preferably cleaves after a Glu or an Asp. Incubation of the prepeptides with $0.1 \mathrm{mg} / \mathrm{mL}$ Glu-C was performed overnight at $37^{\circ} \mathrm{C}$.

Production levels of prenisin, prenisin-(1-22) and prenisin-(1-22) analogs were analyzed via gel electrophoresis. Prepeptides were isolated from the supernatant of minimal medium cell cultures by trichloroacetic acid (TCA) precipitation. The prepeptides were subsequently separated on a tricine-sodium dodecyl sulfate (SDS)-acrylamide gel [24]. Analysis was performed by Coomassie staining (PageBlue ${ }^{\mathrm{TM}}$ ). Prepeptide bands were quantified by measuring the gel band density using ImageJ [25].

Truncated prenisin analogs containing a free C-terminal cysteine were chemically amidated while the prepeptides were bound on a $5 \mathrm{~mL}$ HiTrap SP Sepharose cation-exchange column (GE Healthcare) to prevent multimerization. After loading the prepeptides on the column, the prepeptides were incubated with $10 \mathrm{mg} / \mathrm{mL}$ tris[2-carboxyethyl]phosphine (TCEP) in $5 \mathrm{mM}$ lactate for $30 \mathrm{~min}$ at room temperature to prevent disulfide bond formation between the free cysteines. Subsequently, the prepeptides were incubated with $10 \mathrm{mg} / \mathrm{mL}$ 1-Cyano-4-dimethylaminopyridinium tetrafluoroborate (CDAP) in $5 \mathrm{mM}$ lactate and cyanylation was allowed to take place for 2 hours at room temperature. Cyanylated prepeptides were subsequently eluted from the column with $2 \% \mathrm{NH}_{4} \mathrm{OH}(\mathrm{pH} 10)$. The solution was adjusted to $4 \% \mathrm{NH}_{4} \mathrm{OH}$ (pH 10) for $20 \mathrm{~min}$ at $30{ }^{\circ} \mathrm{C}$ to cleave off the cyanylated Cys and replace the $\mathrm{C}$-terminal negative carboxyl group with a neutral- $\mathrm{CONH}_{2}$. Ammonia was removed by drying the mixture in a speed-vac. Amidation of the peptides was confirmed via mass spectrometry.

Antimicrobial activity of nisin, nisin-(1-22) and nisin-(1-22) analogs was measured against nisin-sensitive L. lactis strains. Indicator strain L. lactis LL108(pORI 280) [18,19] was grown in GM17 containing chloramphenicol $(5 \mu \mathrm{g} / \mathrm{mL})$ and erythromycin $(5 \mu \mathrm{g} / \mathrm{mL})$ to an optical density of 0.1 at $600 \mathrm{~nm}$. After 3-5 hours of incubation in microwell plates with a series of two-fold dilutions of the peptides, growth inhibition was measured at $600 \mathrm{~nm}$. The $50 \%$ inhibitory concentration $\left(\mathrm{IC}_{50}\right)$ was obtained from the midpoint of the sigmoidal growth curve. Halo-forming capacities of (mutant) prenisin-producing L. lactis NZ9000 colonies were measured using an overlay with the NisP-expressing L. lactis pNGnisT pNGnisP strain [20].

Mass spectrometry was performed to confirm that the correct nisin mutants were produced. Samples containing free cysteines were pre-incubated with $1 \mathrm{mg} / \mathrm{mL}$ TCEP to prevent cysteinylation. $1 \mu \mathrm{L}$ of culture supernatant or purified peptide was applied to a target and allowed to dry. Spots from culture supernatants were washed once with $5 \mu \mathrm{L}$ of Millipore water to remove the salts. Spots were subsequently overlayed with $1 \mu \mathrm{L}$ of matrix $(5 \mathrm{mg} / \mathrm{mL} \alpha$-cyano-4-hydroxycinnamic acid in $50 \%$ acetonitrile containing $0.1 \%$ [vol/vol] trifluoroacetic acid). Mass spectra were recorded with a Voyager-DE PRO matrix-assisted laser desorption ionization-time-of-flight (MALDI-TOF) mass 
spectrometer (Applied Biosystems). In order to maintain high sensitivity, an external calibration was applied.

\section{Results and Discussion}

Nisin permeabilizes the membrane by forming transmembrane hybrid pores composed of lipid II and nisin, and inhibits the cell wall synthesis [11-13] by displacing lipid II from the septa [10]. Nisin also auto-induces its own synthesis, inhibits the outgrowth of spores and is recognized by the self-protection mechanisms that provide immunity to producing cells. Previously, we demonstrated that these activities and features could be dissected and separately modulated [8]. In this preceding study we reported on nisin mutants comprising rings $\mathrm{A}, \mathrm{B}$ and $\mathrm{C}$ that are still equipped with antimicrobial activity. Opening of ring $\mathrm{C}$ abolishes activity [26]. Apparently, ring $\mathrm{C}$ is needed for activity, possibly by binding other (truncated) nisin peptides and inducing the segregation of lipid II [1]. The studied nisin-(1-22) and mutants thereof were unable to permeabilize the target membrane but retained significant antimicrobial activity.

\subsection{Ring A Mutants Obtained by Randomization}

Both gallidermin and epidermin possess the same putative lipid II binding motif as the one established for nisin and consist of the same amount of residues as nisin-(1-22) [11,27]. However, even without the capacity to form pores, epidermin and gallidermin were shown to be 10 to 20 times more potent against L. lactis subsp. cremoris HP than nisin [28]. This led us to the idea that the activity of truncated nisin might be optimized via genetic engineering. We randomized the codons for residues 4, 5 and 6 inside ring $A$, and selected five new truncated nisin mutants based on the halo-forming abilities of the colonies. The corresponding nisin-(1-22) mutants showed a high prevalence of phenylalanine and aliphatic amino acids: residues ISL were substituted by VFL, IFL, FFL, LFL and VSL. Growing the colonies in the absence of inducing amounts of nisin revealed that these nisin-(1-22) mutants had lost the capacity to self-induce their synthesis [Figure 2(a,b), first two rows]. In contrast, nisin-(1-22) analogs that contain amino acid substitutions or additions at the C-terminus but no substitutions in ring A are still capable of self-induction [Figure 2(a,b), last row]. Moreover, Rink et al. published several full length nisin mutants with substituted amino acids in ring A that retained the ability to self-induce [8]. Together these data strongly indicate that either an unchanged $\mathrm{N}$ - or C-terminal part of nisin is needed for the capacity of self-induction. By both removing the $\mathrm{C}$-terminal residues 23 to 34 and changing the $\mathrm{N}$-terminal residues under ring $\mathrm{A}$, self-induction was abolished. Analyzing the activity of the purified mutants showed that the $\mathrm{IC}_{50}$ values were not significantly higher than those of nisin-(1-22), as shown by a typical experiment in Table 2. Taken together, these mutants demonstrate that the residues in ring A can be altered without losing antimicrobial activity of nisin-(1-22) or production efficiency [Figure 2(c)]. 
Figure 2. Antimicrobial activity and production of truncated nisin analogs. The three amino acids correspond to residues 4, 5 and 6 and were obtained by genetic randomization and screening for activity. Nisin- is abbreviated to n-. (a) Growth inhibition of L. lactis pNGnisT pNGnisP by colonies producing prenisin-(1-22) ring A mutants (first two rows) and colonies producing truncated prenisin analogs containing C-terminal amino acid substitutions or additions (last row), without nisin induction and (b) with nisin induction. (c) Production levels of prenisin-(1-22) ring A mutants analyzed on a tricine SDS gel. ISL is wild type prenisin-(1-22); wt denotes full length wild type prenisin.

(a)

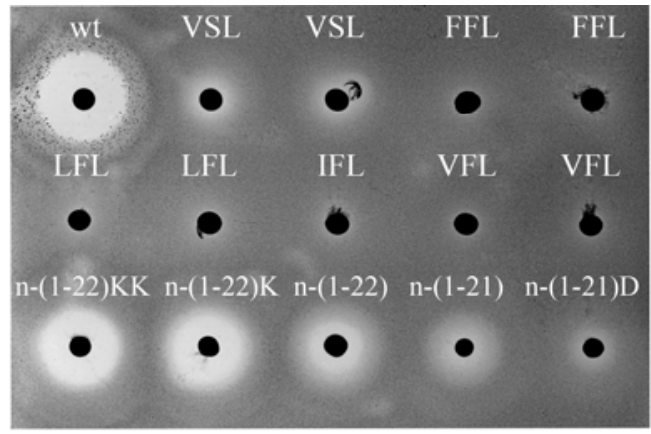

(b)

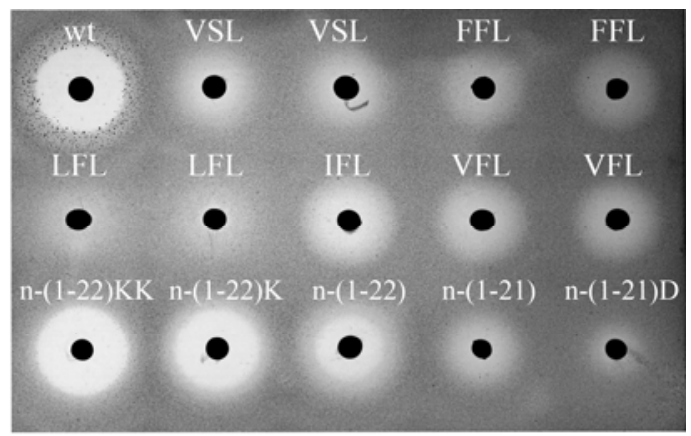

(c)

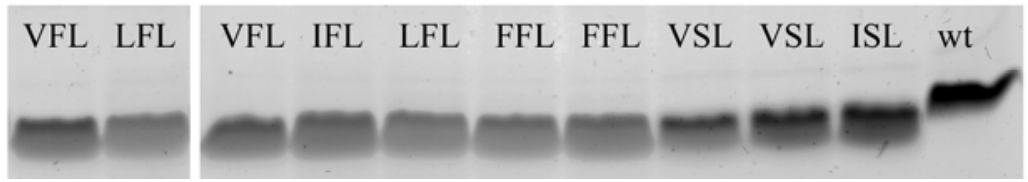

Table 2. Susceptibility of L. lactis LL108(pORI 280) cells to purified nisin and derived mutants.

\begin{tabular}{l|c}
\hline Nisin mutant & Relative IC $_{\mathbf{5 0}}$ values \\
\hline nisin-(1-22) & 1.00 \\
ITSLFLCTPGCKTGALMGCNMK & 0.93 \\
ITSFFLCTPGCKTGALMGCNMK & 0.98 \\
ITSIFLCTPGCKTGALMGCNMK & 1.13 \\
ITSVFLCTPGCKTGALMGCNMK & 0.89 \\
ITSVSLCTPGCKTGALMGCNMK & 1.97 \\
nisin & 0.08 \\
\hline
\end{tabular}

\subsection{Randomization of Residues N20, M21, and K22}

We subsequently randomized the three C-terminal residues of nisin-(1-22): N20, M21, and K22. Based again on the halo size around colonies, we chose 16 active and 12 non-active peptides for further analysis [Figure 3(a)]. Large halos were formed by nisin-(1-22) mutants containing positively charged residues and/or aromatic residues. Most of the mutants that formed no halo contained negatively charged residues and/or prolines. Analyzing the production of the truncated prenisin mutants with tricine SDS gel electrophoresis and Coomassie staining showed that halo size is highly correlated to the quantity of peptide produced [Figure 3(b), Table 3]. An exception is a methionine mutant which is well produced but inactive. Possibly, the C-terminal Met, which is rare in such a position [17], is hindering the formation of ring C. We selected two mutants for a more detailed analysis of their antimicrobial activities: nisin-(1-19)FFF and nisin-(1-19)GLA. After purification of 
these mutants, we performed a comparison with equal concentrations of nisin-(1-22) and full length nisin. Nisin-(1-19)FFF showed a slightly lower activity compared to that of nisin-(1-22) while nisin-(1-19)GLA showed about three-fold lower activity. Halo size is a combined result of antimicrobial activity and production level. In addition, it cannot be excluded that differential diffusion and auto-induction might play a role. Taken together, the randomization and screening resulted in nisin-(1-22) mutants mainly with modulated export instead of modulated intrinsic activity. Evidently, substituting residues N20, M21 and K22 with other amino acids highly impacts production and/or export of nisin-(1-22).

Figure 3. Antimicrobial activity and production of nisin-(1-22) mutants with substituted residues in the C-terminal tail. The three amino acids correspond to residues 20, 21, and 22. (a) Growth inhibition of L. lactis pNGnisT pNGnisP by colonies producing the truncated prenisin mutants, with nisin induction. (b) Production levels of the truncated prenisin mutants visualized on a tricine SDS gel. NMK is wild type prenisin-(1-22); wt denotes full length wild type prenisin.

(a)

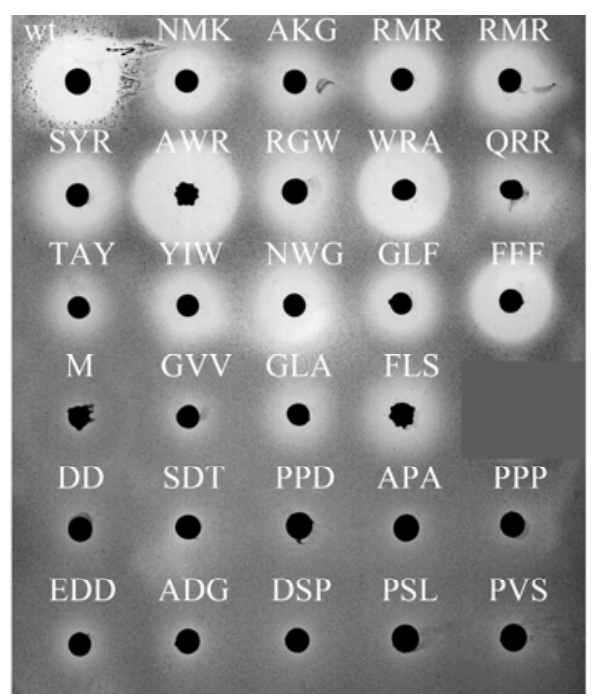

(b)

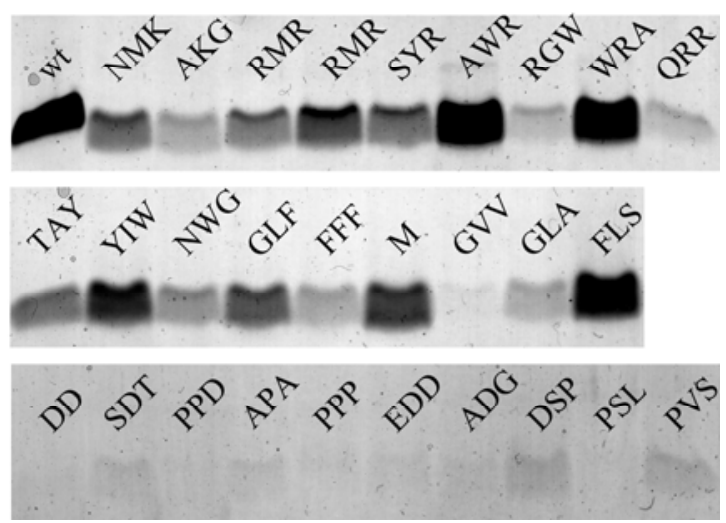

Table 3. Halo size and gel band intensity of nisin-(1-22) mutants with substituted residues in the C-terminal tail. The three amino acids correspond to residues 20, 21, and 22. Values are the average result of three independent experiments, relative to nisin-(1-22).

\begin{tabular}{lcc}
\hline Nisin mutant & Halo size & Band intensity \\
\hline nisin & 1.33 & 1.35 \\
nisin-(1-22) & 1.00 & 1.00 \\
AKG & 0.86 & 0.81 \\
RMR & 1.17 & 1.03 \\
RMR & 1.08 & 1.27 \\
SYR & 1.04 & 1.08 \\
AWR & 1.45 & 1.51 \\
RGW & 0.71 & 0.58 \\
WRA & 1.23 & 1.33 \\
QRR & 0.79 & 0.52 \\
TAY & 0.63 & 0.85 \\
YIW & 0.90 & 1.01 \\
NWG & 1.09 & 0.82 \\
GLF & 1.03 & 0.93 \\
FFF & 1.09 & 0.56 \\
\hline
\end{tabular}


Table 3. Cont.

\begin{tabular}{lcc}
\hline Nisin mutant & Halo size & Band intensity \\
\hline M & 0.00 & 1.05 \\
GVV & 0.37 & 0.31 \\
GLA & 0.75 & 0.75 \\
FLS & 0.94 & 1.20 \\
DD & 0.00 & 0.04 \\
SDT & 0.04 & 0.18 \\
PPD & 0.00 & 0.07 \\
APA & 0.04 & 0.13 \\
PPP & 0.00 & 0.15 \\
EDD & 0.12 & 0.19 \\
ADG & 0.04 & 0.13 \\
DSP & 0.00 & 0.19 \\
PSL & 0.02 & 0.04 \\
PVS & 0.02 & 0.24 \\
\hline
\end{tabular}

\subsection{Engineering Anionic and Cationic Tails in Nisin-(1-22)}

The activity of nisin-(1-22) is about 10-fold higher than that reported for nisin-(1-20) [8,9]. Previously, Rink et al. argued that the relatively high intrinsic antimicrobial activity of nisin-(1-22) might result from the positively charged Lys22 which counterbalances the negative charge of the C-terminal carboxyl group [8]. We therefore aimed to elucidate the effect of a C-terminal positive or negative charge on the activity of truncated nisin. We engineered a series of truncated nisin analogs with a cationic or anionic tail, comprising a $\mathrm{C}$-terminal charge ranging from -2 to +2 . The $\mathrm{C}$-terminal charges were created by genetically engineering additional aspartates or lysines (Table 4).

Table 4. Truncated nisin analogs with different C-terminal charge.

\begin{tabular}{l|c|c|c}
\hline \multicolumn{1}{c|}{ Truncated nisin analog } & $\begin{array}{c}\text { C-terminal } \\
\text { charge }\end{array}$ & $\begin{array}{c}\text { Monoisotopic mass, } \\
\text { calculated (Dalton) }\end{array}$ & $\begin{array}{c}\text { Monoisotopic mass, } \\
\text { measured (Dalton) }\end{array}$ \\
\hline ITSISLCTPGCKTGALMGCNMD & -2 & $2,125.99$ & $2,125.94$ \\
ITSISLCTPGCKTGALMGCNM & -1 & $2,010.96$ & $2,010.80$ \\
ITSISLCTPGCKTGALMGCNMK & 0 & $2,139.06$ & $2,138.86$ \\
ITSISLCTPGCKTGALMGCNMKK & +1 & $2,267.15$ & $2,267.08$ \\
ITSISLCTPGCKTGALMGCNMKKK & +2 & $2,395.25$ & $2,394.97$ \\
ITSISLCTPGCKTGALMGCNMKCK & +1 & $2,370.16$ & $2,370.09$ \\
ITSISLCTPGCKTGALMGCNMKKCK & +2 & $2,498.26$ & $2,497.94$ \\
ITSISLCTPGCKTGALMGCNMK-NH 2 & +1 & $2,138.06$ & $2,138.27$ \\
ITSISLCTPGCKTGALMGCNMKK-NH & +2 & $2,266.15$ & $2,266.01$ \\
\hline
\end{tabular}

Moreover, the highly active lantibiotic epidermin contains a neutral C-terminus through decarboxylation by its decarboxylase EpiD [29]. The absence of C-terminal charges might enhance the antimicrobial activity of nisin-(1-22) even further. Hence, two truncated nisin analogs were engineered with a cysteine-containing tail. This cysteine was subsequently used for chemical amidation of the C-terminus, thus eliminating the negatively charged carboxyl group. 
Amidation is a process widely occurring in nature. About $50 \%$ of all therapeutic peptides are amidated. Lantibiotic enzyme-dehydrated peptides may offer a particular way of amidation since amidation can be obtained by acid-mediated cleavage of dehydroalanines [9]. This method has however some limitations since optimal dehydration of a serine is desirable for sufficient yield and the acid-mediated cleavage should not affect other parts of the molecule. We here chemically amidated truncated nisin ending with an engineered cysteine by preparing an isothiocyanate which is subsequently cleaved off by ammonia, leaving a carboxyl amine. Hence the negatively-charged C-terminal carboxyl group was replaced by a neutral amino group (Table 4, last two mutants).

Prior to the amidation process, we observed multimerization of the truncated nisin analogs with an additional cysteine. Further analyses utilizing trypsin and mass spectrometry indicated that the free cysteine reacts with the dehydrobutyrine at position two or with the dehydroalanine at position five forming an intermolecular lanthionine bond. Mass spectrometry revealed a fragment with a mass that corresponds to the first 12 residues of nisin, ITSISLCTPGCK, with an extra -CK attached. This fragment indicates that the free cysteine at the C-terminal end of these nisin analogs can bind to one of the amino acids in the first 12 residues which is most likely the reactive dehydroalanine at position 5 (Figure 4).

Figure 4. Mass spectrometry analysis of nisin-(1-22)CK digested with trypsin. The fragment with a mass of 1,400 Da (left-hand spectrum) corresponds to the first 12 residues of nisin with an extra - CK attached from the C-terminal end of another nisin peptide. Another fragment (right-hand spectrum) with a mass of 2,389 Da corresponds to residue 1 to 22 with an extra -CK attached.

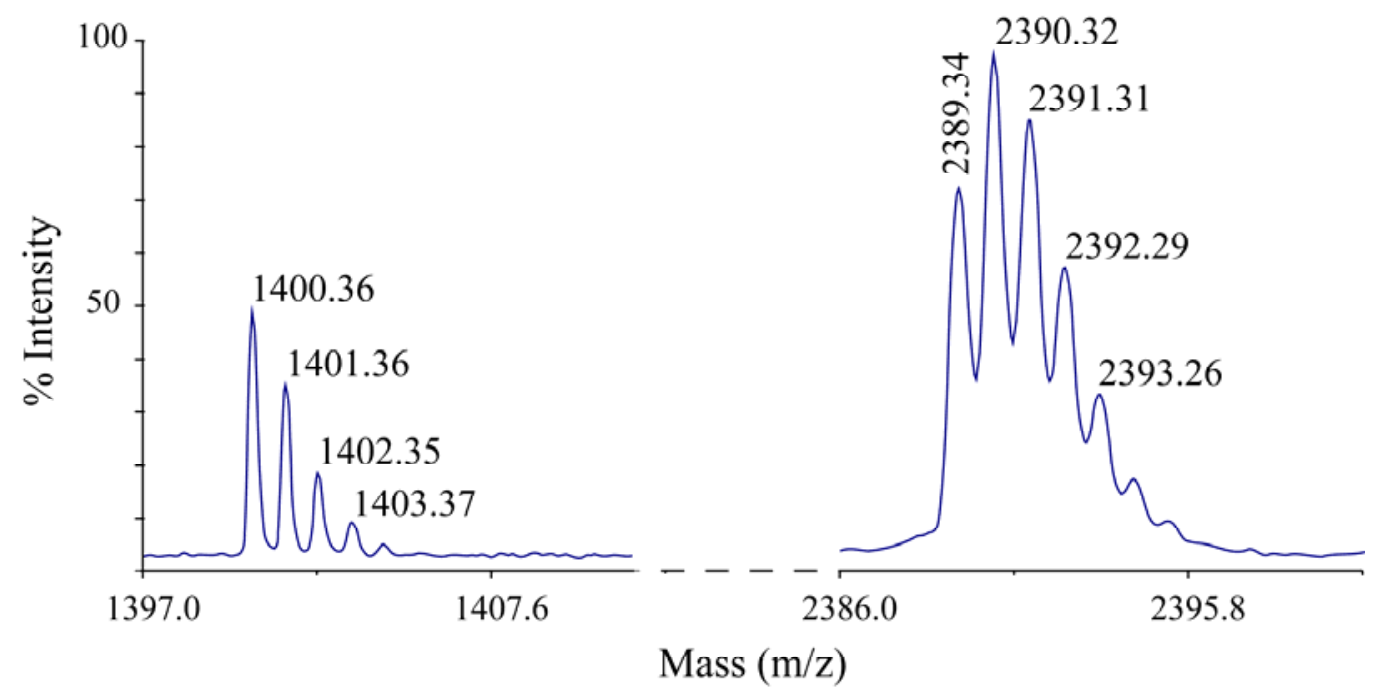

To prevent this multimerization, chemical amidation was performed while the peptides were bound on a cation-exchange column. Isolating the truncated prenisin analogs from supernatant on a cation-exchange column was the first point in the purification process where the peptides were concentrated enough to perform chemical modifications. Furthermore, the bound state of the peptides, next to preventing intermolecular reactions, allows easy removal of chemicals after incubation. 


\subsection{Positive C-Terminal Charge Enhances Antimicrobial Activity of Nisin-(1-22)}

The antimicrobial activity of equal concentrations of the truncated nisin analogs was measured against a nisin-sensitive L. lactis strain [Table 4, Figure 5(a)]. The results demonstrate that the activity of truncated nisin with a neutral or positively charged tail is significantly higher than the activity of truncated nisin with a negatively charged tail. The benefit of additional positive charges levels off since the activity of truncated nisin with two or three C-terminal lysines is not significantly higher than the activity of nisin-(1-22). Also, complete removal of C-terminal charge by amidation of the $\mathrm{C}$-terminus did not result in a higher activity. The production levels of the two negatively charged truncated nisin analogs were lower than those of the neutral or positively charged analogs [Figure 5(b)]. This is consistent with the previous results where substituting residue N20, M21 and/or K22 with a negative residue led to a decreased production level [Figure 3(b)].

Figure 5. Antimicrobial activity and production of truncated (pre)nisin analogs with different C-terminal charge. (a) C-terminal positive charge enhances antimicrobial activity of truncated nisin analogs. Analogs were purified, and equal amounts were tested in a microwell dilution assay. Activities are displayed as the 50\% inhibitory concentration $\left(\mathrm{IC}_{50}\right)$. Apostrophe (') denotes an amidated C-terminus. The C-terminal charge of the peptides is given after the slash. An asterisk indicates significant difference $(P<0.05)$ between the relative antimicrobial activities, as tested by a paired $t$ test. Error bars indicate standard deviations. (b) Production levels of the truncated prenisin analogs visualized on a tricine SDS gel.

(a)

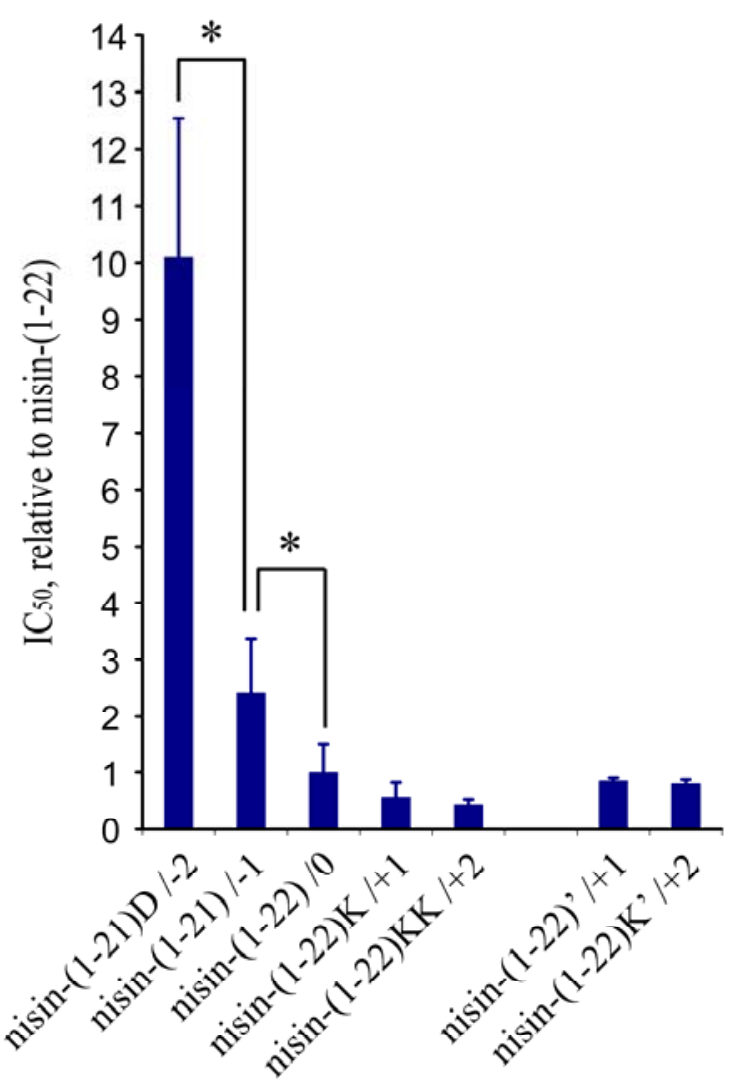

(b)

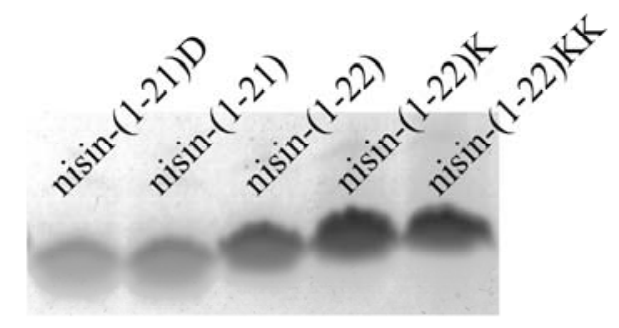


Studies on full length nisin indicated an activity-enhancing effect of positive charge at the C-terminus [30,31]. Most type A lantibiotics are cationic, which appears to enhance their interaction with the negatively charged lipid membrane of the target organism. However, introducing more positively charged amino acids into the C-terminus of nisin did not lead to enhanced antimicrobial activity [30,32,33] and might even hinder pore formation [34]. Analogously, we found that adding more C-terminal lysines did not further increase antimicrobial activity of nisin-(1-22). The susceptibility of target organisms appears to depend on a balanced interaction between the abundance of negatively charged lipids in their membrane and the amount and/or position of charged amino acids in the lantibiotic [33].

\subsection{Mechanistic Aspects of Nisin-(1-22)-Induced Cell Growth Inhibition}

We previously demonstrated that nisin-(1-22) was unable to form pores in the cell membrane of target cells [8]. Therefore its mechanism of cell inhibition likely results from the abduction of lipid II from the septum which attenuates the essential synthesis of the cell wall. Nisin itself inhibits cell growth both by forming pores in the cell membrane and by abduction of lipid II. We here aimed to investigate whether nisin-(1-22) and nisin together might act synergistically or antagonistically. We therefore studied growth inhibition of target cells exposed to different ratios of nisin-(1-22) and nisin. To achieve an antimicrobial activity comparable to full length nisin against a nisin-sensitive L. lactis strain we used ten times higher concentrations of nisin-(1-22). Nisin and nisin-(1-22) were mixed prior to incubation with the nisin-sensitive cells.

Figure 6. (a) Growth inhibition of L. lactis LL108(pORI 280) by nisin-(1-22) and nisin, alone and mixed together. (b) An enlargement of the first half of graph 6a, presented as a contour graph.
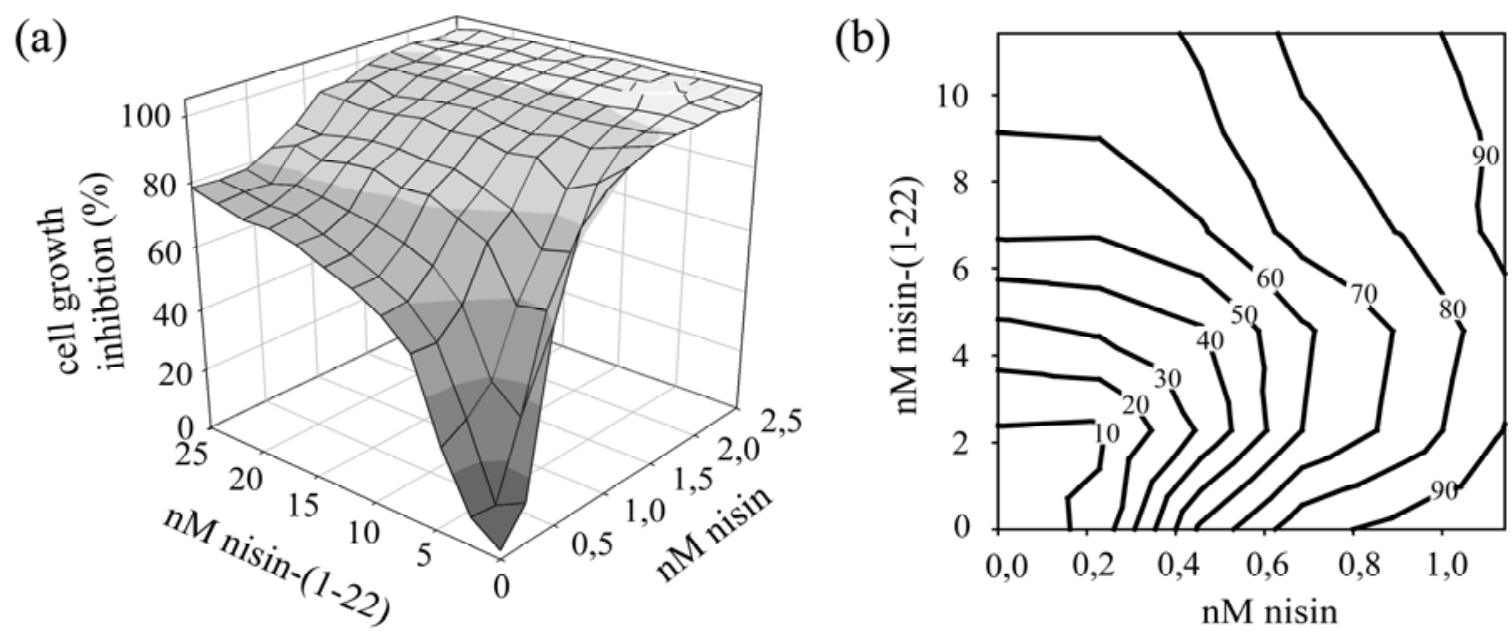

Figure 6 shows the effects of both peptides alone and together on the growth of nisin-sensitive L. lactis. The dynamics of nisin-(1-22) are different from nisin, as evident from the gentler slope of the inhibition curve [Figure 6(a)]. Dissipation of the transmembrane $\mathrm{pH}$ gradient is sufficient for completely arresting cell growth [35]. Therefore the sharp curve obtained with full length nisin likely reflects the benefit of the pore-forming ability of nisin where only a few peptides are needed to form a proton-conducting pore which will swiftly kill the target cell. Adding low concentrations of nisin-(1-22) 
to nisin appeared to inhibit the antimicrobial activity of nisin [Figure 6(b)]. Likely pore formation by a complex of nisin, lipid II and nisin-(1-22) is inefficient due to the presence of nisin-(1-22). At higher concentrations, nisin-(1-22) more or less proportionally enhanced the overall antimicrobial activity.

The mechanistic differences between nisin-(1-22) and nisin likely explain the shapes of the inhibition curves found in this study. Lipid II abduction alone will require more peptide as even a few remaining lipid II molecules might still keep the cell wall growing, albeit at a slower pace. Nisin forms hybrid pores composed of lipid II and nisin. Nisin thereby displays sharper curves and exerts activity at about 10-fold lower concentration than nisin-(1-22). Previously, inhibition of nisin pore formation by nisin-(1-12) has been reported in proteoliposomes [35] and cells [9]. Here nisin-(1-22) inhibited nisin-mediated growth inhibition, depending on the concentration and nisin-(1-22) to nisin ratio. This might be explained by competition for binding to lipid II and/or the formation of nisin-lipid II-nisin-(1-22) complexes which may not, or less efficiently, form pores.

\section{Conclusions}

We demonstrated that the export and activity of truncated nisin can be enhanced by the composition of its C-terminal amino acids, in particular by C-terminal positive charge. Nisin itself is not used as antibiotic for internal use in man. It is degraded in the gut and the dehydroamino acids are unstable at neutral and alkaline $\mathrm{pH}$ [32]. These dehydro amino acids can however be eliminated by engineering, without loss of activity [8] (this study). Small stable nisin analogs may be suitable for oral and pulmonary delivery. Furthermore, their chemical synthesis is less difficult than that of full length nisin and therefore might become economically relevant. On the other hand, the reactivity of these dehydro amino acids might be nicely exploited for the coupling of small lantibiotics to polymers. Different modes of action of antimicrobial peptides may be inspiring for designing antimicrobial polymers. In particular the principle of antimicrobial activity of truncated nisin, which takes place without forming transmembrane pores, might be applicable in polymers. Further understanding of the modulation of activity and/or export efficiency will extend the possibilities to design and engineer improved short lantibiotics with relevant pharmacokinetics and pharmacodynamics or with free dehydroamino acids which enable the coupling to polymers.

\section{References}

1. Gross, E.; Morell, J.L. The Structure of Nisin. J. Am. Chem. Soc. 1971, 93, 4634-4635.

2 Rogers, L.A. The Inhibiting Effect of Streptococcus Lactis on Lactobacillus Bulgaricus. J. Bacteriol. 1928, 16, 321-325.

3 Delves-Broughton, J.; Blackburn, P.; Evans, R.J.; Hugenholtz, J. Applications of the Bacteriocin, Nisin. Anton. Leeuwenhoek 1996, 69, 193-202.

4 Kramer, N.E.; Hasper, H.E.; van den Bogaard, P.T.; Morath, S.; de Kruijff, B.; Hartung, T.; Smid, E.J.; Breukink, E.; Kok, J.; Kuipers, O.P. Increased D-Alanylation of Lipoteichoic Acid and a Thickened Septum are Main Determinants in the Nisin Resistance Mechanism of Lactococcus Lactis. Microbiology 2008, 154, 1755-1762. 
5. Gravesen, A.; Sorensen, K.; Aarestrup, F.M.; Knochel, S. Spontaneous Nisin-Resistant Listeria Monocytogenes Mutants with Increased Expression of a Putative Penicillin-Binding Protein and their Sensitivity to various Antibiotics. Microb. Drug Resist. 2001, 7, 127-135.

6. Lubelski, J.; Rink, R.; Khusainov, R.; Moll, G.N.; Kuipers, O.P. Biosynthesis, Immunity, Regulation, Mode of Action and Engineering of the Model Lantibiotic Nisin. Cell Mol. Life Sci. 2008, 65, 455-476.

7. Schnell, N.; Entian, K.D.; Schneider, U.; Gotz, F.; Zahner, H.; Kellner, R.; Jung, G. Prepeptide Sequence of Epidermin, a Ribosomally Synthesized Antibiotic with Four Sulphide-Rings. Nature 1988, 333, 276-278.

8. Rink, R.; Wierenga, J.; Kuipers, A.; Kluskens, L.D.; Driessen, A.J.; Kuipers, O.P.; Moll, G.N. Dissection and Modulation of the Four Distinct Activities of Nisin by Mutagenesis of Rings A and B and by C-Terminal Truncation. Appl. Environ. Microbiol. 2007, 73, 5809-5816.

9. Chan, W.C.; Leyland, M.; Clark, J.; Dodd, H.M.; Lian, L.Y.; Gasson, M.J.; Bycroft, B.W.; Roberts, G.C. Structure-Activity Relationships in the Peptide Antibiotic Nisin: Antibacterial Activity of Fragments of Nisin. FEBS Lett. 1996, 390, 129-132.

10. Hasper, H.E.; Kramer, N.E.; Smith, J.L.; Hillman, J.D.; Zachariah, C.; Kuipers, O.P.; de Kruijff, B.; Breukink, E. An Alternative Bactericidal Mechanism of Action for Lantibiotic Peptides that Target Lipid II. Science 2006, 313, 1636-1637.

11. Brotz, H.; Josten, M.; Wiedemann, I.; Schneider, U.; Gotz, F.; Bierbaum, G.; Sahl, H.G. Role of Lipid-Bound Peptidoglycan Precursors in the Formation of Pores by Nisin, Epidermin and Other Lantibiotics. Mol. Microbiol. 1998, 30, 317-327.

12. Breukink, E.; Wiedemann, I.; van Kraaij, C.; Kuipers, O.P.; Sahl, H.; de Kruijff, B. Use of the Cell Wall Precursor Lipid II by a Pore-Forming Peptide Antibiotic. Science 1999, 286, 2361-2364.

13. Breukink, E.; van Heusden, H.E.; Vollmerhaus, P.J.; Swiezewska, E.; Brunner, L.; Walker, S.; Heck, A.J.; de Kruijff, B. Lipid II is an Intrinsic Component of the Pore Induced by Nisin in Bacterial Membranes. J. Biol. Chem. 2003, 278, 19898-19903.

14. Hasper, H.E.; de Kruijff, B.; Breukink, E. Assembly and Stability of Nisin-Lipid II Pores. Biochemistry 2004, 43, 11567-11575.

15. Terzaghi, B.E.; Sandine, W.E. Improved Medium for Lactic Streptococci and Their Bacteriophages. Appl. Microbiol. 1975, 29, 807-813.

16. Jensen, P.R.; Hammer, K. Minimal Requirements for Exponential Growth of Lactococcus Lactis. Appl. Environ. Microbiol. 1993, 59, 4363-4366.

17. Rink, R.; Kuipers, A.; de Boef, E.; Leenhouts, K.J.; Driessen, A.J.; Moll, G.N.; Kuipers, O.P. Lantibiotic Structures as Guidelines for the Design of Peptides that can be Modified by Lantibiotic Enzymes. Biochemistry 2005, 44, 8873-8882.

18. Kuipers, O.P.; de Ruyter, P.G.; Kleerebezem, M.; de Vos, W.M. Controlled Overproduction of Proteins by Lactic Acid Bacteria. Trends Biotechnol. 1997, 15, 135-140.

19. Leenhouts, K.; Bolhuis, A.; Venema, G.; Kok, J. Construction of a Food-Grade Multiple-Copy Integration System for Lactococcus Lactis. Appl. Microbiol. Biotechnol. 1998, 49, 417-423. 
20. Kuipers, A.; de Boef, E.; Rink, R.; Fekken, S.; Kluskens, L.D.; Driessen, A.J.; Leenhouts, K.; Kuipers, O.P.; Moll, G.N. NisT, the Transporter of the Lantibiotic Nisin, can Transport Fully Modified, Dehydrated, and Unmodified Prenisin and Fusions of the Leader Peptide with Non-Lantibiotic Peptides. J. Biol. Chem. 2004, 279, 22176-22182.

21. Sambrook, J.; Fritsch, E.F.; Maniatis, T. Molecular Cloning: A Laboratory Manual; Cold Spring Harbor Laboratory Press: Cold Spring Harbor, NY, USA, 1989; p. 545.

22. Holo, H.; Nes, I.F. Transformation of Lactococcus by Electroporation. Methods Mol. Biol. 1995, 47, 195-199.

23. Plat, A.; Kluskens, L.D.; Kuipers, A.; Rink, R.; Moll, G.N. Requirements of the Engineered Leader Peptide of Nisin for Inducing Modification, Export, and Cleavage. Appl. Environ. Microbiol. 2011, 77, 604-611.

24. Schagger, H.; von Jagow, G. Tricine-Sodium Dodecyl Sulfate-Polyacrylamide Gel Electrophoresis for the Separation of Proteins in the Range from 1 to $100 \mathrm{kDa}$. Anal. Biochem. 1987, 166, 368-379.

25. Rasband, W.S. ImageJ; U. S. National Institutes of Health: Bethesda, MA, USA, 1997-2011; Available online: http://imagej.nih.gov/ij/ (accessed on 11 August 2011).

26. Moll, G.N.; Roberts, G.C.; Konings, W.N.; Driessen, A.J. Mechanism of Lantibiotic-Induced Pore-Formation. Antonie Van Leeuwenhoek 1996, 69, 185-191.

27. Hsu, S.T.; Breukink, E.; Tischenko, E.; Lutters, M.A.; de Kruijff, B.; Kaptein, R.; Bonvin, A.M.; van Nuland, N.A. The Nisin-Lipid II Complex Reveals a Pyrophosphate Cage that Provides a Blueprint for Novel Antibiotics. Nat. Struct. Mol. Biol. 2004, 11, 963-967.

28. Bonelli, R.R.; Schneider, T.; Sahl, H.G.; Wiedemann, I. Insights into in vivo Activities of Lantibiotics from Gallidermin and Epidermin Mode-of-Action Studies. Antimicrob. Agents Chemother. 2006, 50, 1449-1457.

29. Kupke, T.; Stevanovic, S.; Sahl, H.G.; Gotz, F. Purification and Characterization of EpiD, a Flavoprotein Involved in the Biosynthesis of the Lantibiotic Epidermin. J. Bacteriol. 1992, 174, 5354-5361.

30. Van Kraaij, C.; Breukink, E.; Rollema, H.S.; Siezen, R.J.; Demel, R.A.; De Kruijff, B.; Kuipers, O.P. Influence of Charge Differences in the C-Terminal Part of Nisin on Antimicrobial Activity and Signaling Capacity. Eur. J. Biochem. 1997, 247, 114-120.

31. Breukink, E.; van Kraaij, C.; Demel, R.A.; Siezen, R.J.; Kuipers, O.P.; de Kruijff, B. The C-Terminal Region of Nisin is Responsible for the Initial Interaction of Nisin with the Target Membrane. Biochemistry 1997, 36, 6968-6976.

32. Rollema, H.S.; Kuipers, O.P.; Both, P.; de Vos, W.M.; Siezen, R.J. Improvement of Solubility and Stability of the Antimicrobial Peptide Nisin by Protein Engineering. Appl. Environ. Microbiol. 1995, 61, 2873-2878.

33. Demel, R.A.; Peelen, T.; Siezen, R.J.; De Kruijff, B.; Kuipers, O.P. Nisin Z, Mutant Nisin Z and Lacticin 481 Interactions with Anionic Lipids Correlate with Antimicrobial Activity. A Monolayer Study. Eur. J. Biochem. 1996, 235, 267-274.

34. van Kraaij, C.; Breukink, E.; Noordermeer, M.A.; Demel, R.A.; Siezen, R.J.; Kuipers, O.P.; de Kruijff, B. Pore Formation by Nisin Involves Translocation of Its C-Terminal Part across the Membrane. Biochemistry 1998, 37, 16033-16040. 
35. Moll, G.N.; Clark, J.; Chan, W.C.; Bycroft, B.W.; Roberts, G.C.; Konings, W.N.; Driessen, A.J. Role of Transmembrane $\mathrm{pH}$ Gradient and Membrane Binding in Nisin Pore Formation. J. Bacteriol. 1997, 179, 135-140.

(C) 2011 by the authors; licensee MDPI, Basel, Switzerland. This article is an open access article distributed under the terms and conditions of the Creative Commons Attribution license (http://creativecommons.org/licenses/by/3.0/). 